\title{
Uma abordagem cognitiva sobre 0 processo de institucionalização de sistemas locais de produção e inovação
}

\author{
Cláudio Roberto Leandro ${ }^{a *}$, João Amato Neto ${ }^{b}$ \\ ${ }^{a *}$ crleandro@usp.br, USP, Brasil \\ bamato@usp.br, USP, Brasil
}

\begin{abstract}
Resumo
A evolução de um Sistema Local de Produção e Inovação (SLPI) é acompanhada pela formação de arranjos institucionais responsáveis pela coordenação das ações entre empresários e demais agentes locais. 0 período embrionário desse processo é marcado por dificuldades em alinhar as instituições e as regras sociais às regras individuais determinadas pela lógica empresarial. 0 artigo analisa como o nível de habilidade cognitiva empresarial influencia a capacidade de interpretação das instituições presentes em um SLPl. A pesquisa, desenvolvida com 20 empresários em uma aglomeração do setor de vestuário na cidade de Indaiatuba, propõe uma metodologia para mensurar a correlação entre a variação nos níveis de habilidade cognitiva dos empresários e a capacidade de interpretação das instituições. A pesquisa evidencia que existem diferentes níveis de estruturas cognitivas dentro do SLPI e que a variação nos níveis de cognição empresarial está associada à capacidade de interpretação das instituições.
\end{abstract}

Palavras-chave

Instituições. Empreendedorismo. Cognição empresarial. Sistemas locais de produção e inovação.

\section{Introdução}

Os Sistemas Locais de Produção e Inovação (SLPI) são reconhecidos pela capacidade de elevação da competitividade de micro, pequenas e médias empresas. Para Amato Neto (2009), esse resultado é alcançado pela proximidade entre empresas, universidades, centros tecnológicos, poder público e associações de classe. 0 autor complementa que a proximidade entre esses agentes contribui para a geração e disseminação de recursos produtivos, tecnológicos e mercadológicos.

A eficácia das ações coletivas é dependente de regras, normas e crenças que são responsáveis pela coordenação do comportamento dos indivíduos (Amato Neto, 2009; Belussi et al., 2008; Amato Neto, 2005). Em SLPls maduros, é possível evidenciar um padrão de comportamento bem disseminado entre os agentes locais. Para esses agentes, o desempenho individual está associado à manutenção da estrutura e da reputação local (Belussi et al., 2008). Em SLPls embrionários, existe apenas a proximidade geográfica entre os agentes com elos fracos e padrões divergentes de comportamento (Amato Neto, 2009). Nesse estágio, as regras, normas e crenças não são interpretadas da mesma forma pelos agentes locais. Os conflitos e as ações isoladas são comuns e prevalecem apenas as ações individuais em detrimento de uma estrutura cooperada com vistas à manutenção da reputação e da tradição local (Leandro \& Amato Neto, 2007).

A intenção dos agentes locais de promoverem a evolução dos SLPls exige a configuração de uma estrutura social com regras, normas e crenças aceitas e enraizadas nas práticas individuais. Mas mudanças institucionais e individuais não acontecem ao mesmo tempo (Stephan \& Uhlaner, 2010). Os indivíduos podem compreender com mais facilidade as transformações sociais em função da estrutura cognitiva que possuem (Leddo \& Abelson, 1986). Para Billet (1996), os indivíduos aprendem com a estrutura social ao tentarem resolver as contradições, impasses e os problemas dentro de um determinado domínio. A compreensão das relações entre regras 
sociais (instituições) e regras individuais (estrutura cognitiva) é dependente da convergência de ambas para um mesmo domínio.

0 domínio utilizado nesse trabalho para estudar as relações sociais e individuais é a atividade empreendedora ou a capacidade que as duas estruturas têm de mobilizarem recursos produtivos, tecnológicos e mercadológicos para promover o desenvolvimento de novos negócios. Dentro desse domínio, o objetivo do artigo é analisar como as estruturas cognitivas influenciam a capacidade empresarial para interpretar as instituições.

Para alcançar esse objetivo, o artigo desenvolve uma revisão da literatura sobre as instituições, a cognição empresarial e sua relação com a atividade empreendedora. Na sequência, um modelo conceitual é desenvolvido para mensurar a correlação entre a variação do nível de habilidades cognitivas empresariais e sua capacidade de interpretar as instituições. Esse modelo foi aplicado a 20 empresas pertencentes ao $\mathrm{SLPl}$, em estágio embrionário, do setor de vestuário localizado na cidade de Indaiatuba, interior do estado de São Paulo. 0 artigo é finalizado com a análise dos dados e a as considerações finais.

\section{Revisão da literatura}

\subsection{Instituições e a atividade empreendedora}

0 empreendedorismo está associado à capacidade de os indivíduos iniciarem processos de busca de novas oportunidades, gerarem ideias, alocarem recursos para converter as ideias em produtos e disseminarem os produtos no mercado consumidor. Embora a literatura possa tratar a atitude empreendedora como vocação individual, Baumol (1990) argumenta que as diferenças econômicas entre países e regiões não é explicada pela natureza empreendedora dos indivíduos, mas pela forma como essa natureza é canalizada dentro de um sistema econômico.

Esse canal representa uma estrutura social capaz de estimular ou restringir a ação dos empreendedores (Hofstede et al., 2010; Lepak et al., 2007; Meyer et al., 2009). Para Acs et al. (2009), os empresários, ao desenvolverem novos negócios, precisam proteger suas criações por patentes, precisam de leis e fóruns de julgamento para reforçarem seus contratos de relacionamento e também precisam acreditar que o poder público possui limites sobre a tributação. Hessels et al. (2008) argumentam que a atividade empreendedora está associada à regulação técnica, concorrencial e econômica das nações. Aidis et al. (2009) e Bowen e DeClerq (2008) complementam que as ações empreendedoras também dependem de acesso a fontes de financiamento e de uma infraestrutura educacional eficiente. Para Greif (2006) e North (1990), a complexidade institucional atua como um sistema de informação compartilhado que contribui para a redução da incerteza e para o estímulo da atividade empreendedora.

\subsubsection{Como as instituições se vinculam aos indivíduos}

Para Bruton et al. (2010), o desenvolvimento da teoria institucional é fundamentado nas forças da estrutura regulatória, social e cultural que envolve uma sociedade. Essas forças são discutidas por diferentes escolas como a escola econômica, representada por North (1990); pela sociologia (DiMaggio \& Powell, 1983); teoria das organizações (Meyer \& Rowman, 1991); e ciência política (Shepsle \& Bonchek, 1996).

Scott (2007) faz uma contribuição relevante para o entendimento das instituições ao construir uma estrutura analítica compilando as forças institucionais estudadas pela economia, sociologia, ciência política e pela teoria das organizações. 0 trabalho de Scott (2007) conclui que as instituições possuem três categorias de forças que o autor classifica como pilares ou dimensões.

0 primeiro pilar, denominado regulatório, deriva da escola econômica e representa a estrutura legal que coordena as ações dos indivíduos. Dentro do domínio discutido nesse trabalho, alguns elementos podem ser destacados, como os acordos setoriais, padrões técnicos e tecnológicos ou, ainda, esse pilar pode ser entendido como a regulação econômica, técnica e concorrencial que rege um sistema econômico.

0 segundo pilar, social e normativo, é definido por valores e normas compartilhados pelos indivíduos. Os valores definem o que é considerado apropriado para uma sociedade ou comunidade e as normas determinam como as coisas devem ser feitas consistentemente com os valores. 0 pilar normativo é relevante para um SLPI, pois representa as conexões sociais entre os diversos agentes, as normas locais que determinam o padrão de cooperação e o compartilhamento de recursos produtivos, tecnológicos e mercadológicos necessários para a construção de uma reputação local.

0 terceiro pilar, cultural e cognitivo, representa um modelo mental compartilhado por uma sociedade, o senso comum ou o inconsciente dos indivíduos. Esse pilar é fortemente influenciado pela cultura e, no contexto dos negócios, representa a valorização da sociedade pela atividade empreendedora. Hofstede (1980) e Hofstede et al. (2010) argumentam que o padrão cultural de uma sociedade é influenciado por cinco fatores: 
- Distância do poder: Representa a tolerância de uma sociedade à desigualdade do poder. Sociedades com alto distanciamento atribuem a atividade empreendedora apenas a grupos sociais restritos e o poder é distribuído de forma desigual.

- Intolerância à incerteza: Algumas sociedades necessitam de regras claras e bem definidas e se sentem mais confortáveis apenas em situações familiares. Situações não estruturadas que exigem levantamento de hipóteses, investigação, cognição, inferências e análise de riscos não são geralmente bem aceitas.

- Orientação para o longo prazo: Sociedades orientadas para o longo prazo têm foco na perseverança e busca por resultados. Sociedades voltadas para o curto prazo valorizam a tradição e o cumprimento das regras sociais.

- Gênero (masculino/feminino): Sociedades com valores masculinos têm foco na competitividade e assertividade, enquanto as sociedades com gênero mais feminino possuem foco na cautela e na modéstia.

- Individualismo: Sociedades mais individualistas possuem elos sociais fracos e a formação de grupos sociais é limitada. As sociedades mais coletivas possuem a lealdade como um fator básico.

As dimensões apresentadas por Hofstede (1980) e Hofstede et al. (2010) podem trazer implicações para os negócios como, por exemplo, a intolerância à incerteza. Novos negócios oferecem situações ambíguas, não estruturadas, e exigem dos indivíduos alguma capacidade para reconhecer os elementos que compõem a incerteza.

As três dimensões institucionais determinam as regras, normas sociais e as crenças que conduzem as ações dentro da atividade empresarial (Bello et al., 2004). Baumol (1990) e Stephan \& Uhlaner (2010) argumentam que os países e as regiões divergem quanto à forma de construir as instituições. Stephan \& Uhlaner (2010) ainda complementam que algumas sociedades conseguem estimular novos empreendimentos apostando na competência individual dos empresários, enquanto outras, para alcançar o mesmo resultado, precisam desenvolver uma intensa estrutura de apoio social.

\subsection{Cognição do empresário}

Os estudos de Leddo \& Abelson (1986) evidenciam que os indivíduos, em um determinado domínio, possuem estruturas cognitivas próprias como, por exemplo, jogadores de xadrez, programadores de computador, legisladores e empresários. Os autores complementam que essas estruturas permitem que os indivíduos absorvam e processem informações de forma diferenciada em comparação com indivíduos não especialistas. McGee et al. (2009), Busenitz (2007) e Bello et al. (2004) complementam que os indivíduos com estruturas ou roteiros cognitivos especialistas reconhecem e processam informações rapidamente, enquanto um indivíduo inexperiente dependeria de muito esforço para alcançar o mesmo resultado.

Mitchell et al. (2000) evidenciam que os empresários tomam decisões sobre novos negócios com a utilização de roteiros ou scripts. Esses roteiros representam estruturas de conhecimento que são acionadas para avaliar e desenvolver novos negócios. Várias contribuições evidenciam na literatura (Holm, 1995; Glaser, 1990; Mitchell et al., 2000; Baron, 2004; Corbett \& Hmieleski, 2007) o papel dos roteiros no comprometimento dos empresários com o desenvolver de novos negócios.

As estruturas ou roteiros cognitivos, segundo Leddo \& Abelson (1986), são influenciados por cinco fatores:

- Características próprias dos indivíduos;

- Experiência acumulada;

- Acesso a e posse de recursos;

- Conhecimento específico em determinada área;

- Educação recebida anteriormente.

Os roteiros auxiliam os empresários nas decisões devido à natureza sequencial que um roteiro possui e à presença de normas que guiam as ações dos indivíduos dentro de uma área específica. Para Leddo \& Abelson (1986), os especialistas possuem, pelo menos, um conjunto de três roteiros.

0 primeiro roteiro, denominado de entrada, é utilizado para avaliar o contexto antes de agir. 0 segundo roteiro se refere às aspirações dos indivíduos e se traduz na prontidão, disposição e na inclinação de aplicar o conhecimento em uma determinada finalidade. 0 terceiro roteiro, centrado nas habilidades, contempla o conhecimento (técnicas, habilidades e competências específicas) que um indivíduo possui dentro da área de domínio. Leddo \& Abelson (1986) complementam que o primeiro roteiro se refere à posse de conhecimento sequencial para avaliar a entrada em uma situação enquanto os roteiros relacionados às aspirações e habilidades permitem a realização (fazer, aplicar) de um objetivo.

No contexto empresarial, Mitchell et al. (2000) reproduzem o modelo discutido por Leddo \& Abelson (1986) para o desenvolvimento de negócios. Para os autores, os roteiros de entrada, de aspirações e de habilidades podem ser estruturados para a ação empresarial da seguinte forma:

- Roteiros de entrada: Proporcionam a capacidade de processamento de informação para avaliar a 
potencialidade de um novo negócio. Esses roteiros apresentam a seguinte estrutura:

- Proteção de ideias: Os empresários avaliam a possibilidade de proteger o negócio contra imitações (marcas, patentes, segredos industriais, franquias ou contratos).

- Redes sociais: Novos negócios exigem contatos, relacionamentos, fontes de informações que possam ampliar a base de conhecimento sobre as oportunidades.

- Acesso a recursos: A entrada em um novo negócio depende dos recursos financeiros, humanos, produtivos e tecnológicos que o empresário possui.

- Habilidade para avaliar um novo negócio: Os empresários precisam de conhecimentos específicos para analisar investimentos e riscos, dimensionar o mercado e difundir as suas criações pelos canais de distribuição.

- Roteiros de aspiração: Novos negócios oferecem obstáculos e os roteiros de aspiração se referem à estrutura de conhecimento que os empresários possuem para ajuste e manutenção da trajetória. Esses roteiros se desdobram em três:

- Foco no processo de busca: A informação precisa ser extraída e compilada de várias fontes. A busca por informações persiste até que todos os elementos envolvidos no desenvolvimento do negócio sejam encontrados e avaliados.

- Motivação sobre uma oportunidade: A consolidação de um negócio depende da capacidade de o empresário identificar e diferenciar os sinais e corrigir a trajetória. Os empresários centrados na criação de um negócio preferem tentar e falhar que perder uma oportunidade.

- Tolerância à incerteza: A atividade empreendedora exige do indivíduo afinidade com novas situações ou áreas desconhecidas. Esses ambientes desconhecidos precisam ser interpretados com seus elementos de risco.

- Roteiros de habilidade: Esses roteiros consistem no conhecimento específico que os empresários possuem para desenvolver um negócio. Esses roteiros estão subdivididos em:

- Diagnóstico: Capacidade empresarial para decompor e sistematizar os elementos necessários para a criação de um empreendimento.

- Conhecimento situacional: As experiências acumuladas pelos empresários geram lições que retroalimentam as decisões em novas situações. Nesse momento, a aprendizagem com negócios anteriores é resgatada, comparada e reproduzida em um novo contexto.

- Adequação da capacidade à oportunidade: Recursos, habilidades, competências e atitudes são analisados e adequados a novas oportunidades.
Leddo \& Abelson (1986) complementam que os três roteiros (configuração de forças) se transformam em uma estrutura cognitiva especialista. Empresários com limitações no roteiro de aspirações podem apresentar baixa motivação para desenvolver novos negócios. Limitações nos roteiros de habilidades podem implicar em dificuldades para explorar os roteiros de entrada.

Os roteiros cognitivos permitem compreender como os empresários pensam ou como as regras individuais são estruturadas (Baron, 2004; Anderson, 1993). Esses roteiros remetem os empresários para um ambiente institucional onde a atividade empreendedora pode ser estimulada ou restringida. A forma como os roteiros cognitivos influenciam a capacidade do empresário para interpretar as instituições é o objeto de estudo deste trabalho e será discutida nas próximas seções.

\section{Construção do modelo conceitual}

Pesquisas de diversos autores oferecem evidências sobre a relação entre as instituições e a atividade empreendedora (Acs, 2009; Smith et al., 2009; Peng et al., 2008; Manolova et al., 2008; Meyer et al., 2009, Lim et al., 2010). Embora essa relação seja amplamente explorada pela literatura, os estudos sobre a forma como as instituições se associam à estrutura cognitiva dos empresários ainda são poucos (Lim et al., 2010; Mitchell et al., 2000).

0 processo de institucionalização de um SLPI depende da capacidade de os empresários interpretarem como as regras sociais criam corredores para a atividade empreendedora. Para Billet (1996), as regras sociais se alinham às regras individuais pela resolução de problemas. Para o autor, ao resolver um problema, os indivíduos resgatam o conhecimento acumulado e o confrontam com a estrutura social. 0 domínio utilizado neste artigo é a atividade empreendedora. Esse domínio é avaliado em duas dimensões. A primeira, endógena, refere-se à capacidade de o empresário desenvolver novos produtos, novos processos, novos mercados ou novas formas de organização (Schumpeter, 1934). Essa capacidade está relacionada à posse de uma estrutura cognitiva específica para a ação empresarial (Leddo \& Abbelson, 1986; Mitchell et al., 2007). A segunda dimensão, exógena, refere-se à estrutura institucional que permeia toda a ação empresarial (Scott, 2007; Baumol, 1990). Para analisar a relação entre a capacidade do empresário para interpretar as instituições e a estrutura cognitiva, as seguintes proposições serão analisadas:

- Proposição 1: Empresários que possuem roteiros cognitivos de entrada, aspiração e habilidades reconhecem os elementos institucionais do ambiente regulatório, social e normativo e cultural e cognitivo. 
Empresários que possuem roteiros cognitivos relacionados à entrada, aspiração e habilidades têm um nível de experiência maior sobre o desenvolvimento de negócios e espera-se que tenham, também, uma capacidade maior para identificar os elementos institucionais que influenciam a atividade empreendedora. Para os empresários com limitações nos roteiros cognitivos, pressupõe-se dificuldade para reconhecer os elementos institucionais.

- Proposição 2: Empresários que possuem roteiros cognitivos de entrada, aspiração e habilidades reconhecem o nivel de qualidade dos elementos institucionais quanto à sua capacidade para estimular ou restringir o desenvolvimento de novos negócios.

Os empresários que possuem roteiros cognitivos precisam reconhecer os elementos institucionais, além disso, espera-se que também sejam capazes de avaliar o nível de qualidade desses elementos. Qualidade significa a capacidade de redução dos custos de transação.

- Proposição 3: Empresários que reconhecem os elementos institucionais e seus níveis de qualidade são propensos a interagir com o ambiente institucional.

Os empresários que reconhecem os elementos institucionais e seus níveis de qualidade têm mais facilidade para incorporar as instituições ou desenvolver projetos de mudança nas regras sociais.

Para a avaliação das proposições, o modelo de pesquisa foi desdobrado em três conjuntos de variáveis: controle, estrutura institucional e cognição empresarial.

\subsection{Variáveis de controle}

O objetivo do artigo é analisar se a cognição empresarial está associada à capacidade do empresário interpretar as instituições. Para alcançar esse objetivo, as duas dimensões (social e individual) são construídas sobre um único domínio e precisam estar isentas da influência de outras variáveis. As seguintes variáveis de controle foram consideradas sobre os empresários pesquisados:

- Desenvolvimento de um negócio que esteja em operação;

- Tempo de permanência como principal gestor da empresa;

- Tempo de permanência na região;

- Experiência anterior como gestor;

- Tempo de educação formal;

- Idade.
Essas variáveis de controle asseguram que a pesquisa seja desenvolvida sobre empresários que (1) tenham experiência sobre, pelo menos, uma empresa em operação; (11) estejam pelo menos há três anos à frente dos negócios; (111) estejam pelo menos há três anos na região do SLPI; (IV) possuam experiência anterior como empresário ou gestor; (V) tempo de educação formal; e (VI) idade. Essas variáveis são relevantes para controlar os fatores relacionados à experiência acumulada nos negócios, em contato com a região e em contato com o sistema educacional.

\subsection{Estrutura institucional e cognição empresarial}

As variáveis referentes à estrutura institucional se desdobram em três níveis, conforme a Tabela 1.

As variáveis referentes aos roteiros cognitivos empresariais são apresentadas na Tabela 2 .

Os três conjuntos de variáveis compõem o modelo de pesquisa para analisar as proposições e alcançar o objetivo proposto neste trabalho.

\section{Metodologia}

\subsection{Amostra}

O SLPI estudado neste trabalho pertence ao setor de vestuário, mais especificamente à confecções de roupas jeans na cidade de Indaiatuba, interior do Estado de São Paulo. Foram selecionados 20 empresários de micro e pequenas empresas para participar da pesquisa. Em 2007, a prefeitura iniciou um processo de formação de um SLPI com a participação dos empresários locais, do sindicato das confecções, das universidades, do SEBRAE e da Associação das Empresas de Confecções. A proposta do SLPI foi estimular a atividade empresarial e recuperar a reputação de capital nacional do jeans. 0 processo de formação do SLPI tem recebido atenção e adesão de poucos empresários, o que mantém o SLPl em um estágio embrionário.

\subsection{Unidade de medida das variáveis de pesquisa}

A unidade de análise é o próprio empresário, com seus roteiros cognitivos e sua capacidade de interpretação das instituições. 0 domínio utilizado é a atividade empreendedora que compreende o lançamento de produtos, acesso a novos mercados, novos clientes ou até mesmo a ampliação da capacidade produtiva. 
Tabela 1. Variáveis do ambiente institucional.

\begin{tabular}{|c|c|c|c|}
\hline Nível & Variável & Relação com a atividade empreendedora & Principais autores \\
\hline \multirow[t]{4}{*}{ Regulatório } & Sistema financeiro & Disponibilidade, facilidade, prazo e custo do capital & $\begin{array}{l}\text { North (1990); Lim et al. } \\
(2010)\end{array}$ \\
\hline & Sistema educacional & Educação em negócios e oferta de mão de obra qualificada & $\begin{array}{l}\text { Acs et al. (2009); Peng et al. } \\
\text { (2008) }\end{array}$ \\
\hline & Sistema legal & $\begin{array}{l}\text { Propriedade intelectual, regulação técnica, concorrencial e } \\
\text { econômica, estrutura burocrática e legislação tributária }\end{array}$ & $\begin{array}{l}\text { North (1990); Bruton et al. } \\
(2010)\end{array}$ \\
\hline & Relações de confiança & Nível de confiança na infraestrutura legal & North (1990) \\
\hline \multirow[t]{3}{*}{$\begin{array}{l}\text { Social e } \\
\text { normativo }\end{array}$} & $\begin{array}{l}\text { Visão, inovação e } \\
\text { criatividade }\end{array}$ & Valorização do pensamento criativo (geração de ideias) & Marquis \& Battillana (2009) \\
\hline & Conectividade social & $\begin{array}{l}\text { Valorização da busca e do compartilhamento de informações e } \\
\text { recursos }\end{array}$ & $\begin{array}{l}\text { Belussi et al. (2008); Amato } \\
\text { Neto (2009) }\end{array}$ \\
\hline & Reputação & Valorização da marca regional como diferencial & Amato Neto (2009) \\
\hline \multirow[t]{5}{*}{$\begin{array}{l}\text { Cultural e } \\
\text { cognitivo }\end{array}$} & Individualismo & Tendência por decisões isoladas & $\begin{array}{l}\text { Hofstede (1980); } \\
\text { Hofstede et al. (2010) }\end{array}$ \\
\hline & Intolerância à incerteza & $\begin{array}{l}\text { Necessidade de regras claras e bem definidas e preferências por } \\
\text { ambientes familiares }\end{array}$ & $\begin{array}{l}\text { Hofstede (1980); } \\
\text { Hofstede et al. (2010) }\end{array}$ \\
\hline & $\begin{array}{l}\text { Orientação ao longo } \\
\text { Prazo }\end{array}$ & Foco na perseverança & $\begin{array}{l}\text { Hofstede (1980; } \\
\text { Hofstede et al. (2010) }\end{array}$ \\
\hline & Gênero masc./fem. & Foco na competitividade & $\begin{array}{l}\text { Hofstede (1980); } \\
\text { Hofstede et al. (2010) }\end{array}$ \\
\hline & Distância do poder & Tolerância às diferenças no acesso a recursos públicos & $\begin{array}{l}\text { Hofstede (1980); } \\
\text { Hofstede et al. (2010) }\end{array}$ \\
\hline
\end{tabular}

Fonte: autores.

\subsection{Coleta de dados}

Um protocolo de pesquisa foi desenvolvido para o estudo de caso com as variáveis apresentadas nas Tabelas 1 e 2 e as variáveis de controle. Para cada variável foi definido um roteiro com diretrizes e questões indicativas para obtenção dos dados necessários. 0 protocolo também apresenta as fontes de obtenção dos dados. As fontes de dados foram entrevistas com os empresários dentro das empresas e em reuniões promovidas com os demais agentes locais. As entrevistas foram desenvolvidas com questões semiestruturadas e cada empresário ofereceu informações sobre sua história profissional frente à empresa que administra. As respostas foram confrontadas com a linha de produtos, os processos produtivos e o modelo de negócio adotado por cada um. Um teste piloto foi desenvolvido em duas empresas para verificar a estrutura dos questionários. Alguns problemas foram detectados quanto à interpretação das questões e foram necessárias modificações quanto à exposição. Para facilitar a interpretação, as questões foram apresentadas com opções contraditórias. Por exemplo, para avaliar se o empresário se preocupa com o processo de busca de novas oportunidades, as questões foram colocadas da seguinte forma: "qual é a prioridade: buscar novas oportunidades para aplicar os seus recursos ou buscar novas formas de gerenciar os recursos?” Essa forma de exposição permitiu que cada empresário descrevesse suas ações dentro da estrutura teórica apresentada pelo presente trabalho.

\subsection{Critérios adotados para reduzir dados}

0 processo de redução de dados tem foco na caracterização de cada empresário frente às variáveis de controle, institucionais e de cognição empresarial. A redução foi desenvolvida em dois estágios. 0 primeiro estágio se concentra na organização e codificação dos dados, de acordo com o protocolo de pesquisa. 0 segundo estágio se concentra na mensuração das variáveis apresentadas nas Tabelas 1 e 2 para cada empresário. As variáveis de controle são avaliadas também para cada empresário e, posteriormente, associadas com as práticas institucionais e a estrutura cognitiva.

As variáveis referentes às práticas institucionais são avaliadas como regras sociais compreendidas e aceitas pelos empresários. Os dados obtidos são classificados em três níveis.

0 primeiro nível, elevado, se refere ao reconhecimento dos elementos institucionais mencionados na Tabela 1 para o desenvolvimento dos negócios. Nesse nível, por exemplo, o empresário consegue associar a importância das leis de proteção de patente ou a tradição local com a necessidade de proteção de ideias. Ao associar o elemento institucional com o elemento cognitivo, o empresário também 
Tabela 2. Variáveis da cognição do empresário.

\begin{tabular}{|c|c|c|c|}
\hline Roteiro cognitivo & Variável & Foco & Principais autores \\
\hline \multirow[t]{4}{*}{ Entrada } & Apropriação de ideias & Mecanismos utilizados para proteção contra imitação & Leddo \& Abelson (1986) \\
\hline & Acesso e posse de recursos & $\begin{array}{l}\text { Posse de recursos tangíveis e intangíveis para } \\
\text { desenvolver negócios }\end{array}$ & Alexander (2004) \\
\hline & Redes de negócios & $\begin{array}{l}\text { Contatos e relacionamento com agentes que facilitem o } \\
\text { desenvolvimento de novos negócios }\end{array}$ & Busenitz \& Lau (1997) \\
\hline & $\begin{array}{l}\text { Habilidades para } \\
\text { desenvolver negócios }\end{array}$ & Conhecimentos em pesquisar mercado & Mitchell et al. (2000) \\
\hline \multirow[t]{3}{*}{ Aspiração } & Foco no processo de busca & $\begin{array}{l}\text { Atenção constante no processo de busca de } \\
\text { oportunidades }\end{array}$ & Mitchell et al. (2000) \\
\hline & $\begin{array}{l}\text { Motivação pela } \\
\text { oportunidade }\end{array}$ & $\begin{array}{l}\text { Capacidade de superar os obstáculos durante o } \\
\text { desenvolvimento de negócios }\end{array}$ & Mitchell et al. (2000) \\
\hline & Tolerância à incerteza & Capacidade de identificar e gerenciar elementos de risco & Mitchell et al. (2000) \\
\hline \multirow[t]{3}{*}{ Habilidades } & Diagnóstico & $\begin{array}{l}\text { Capacidade de decompor os elementos para desenvolver } \\
\text { negócios }\end{array}$ & Mitchell et al. (2000) \\
\hline & Situacional & $\begin{array}{l}\text { Capacidade de incorporar a experiência em novos } \\
\text { contextos }\end{array}$ & Mitchell et al. (2000) \\
\hline & Ajuste & Capacidade de adequar a capacidade à oportunidade & Mitchell et al. (2000) \\
\hline
\end{tabular}

Fonte: autores.

deve avaliar o nível de qualidade desses elementos. A qualidade se refere à capacidade de um elemento institucional reduzir os custos de transação.

No segundo nível, intermediário, os empresários têm um reconhecimento parcial (pelo menos 50\%) dos elementos de cada uma das dimensões institucionais.

No terceiro nível, baixo, os empresários têm dificuldades para reconhecer os elementos institucionais e seu nível de qualidade (abaixo de 50\%).

Os três níveis evidenciam a aderência às regras sociais incorporadas pelos empresários (elevado, intermediário e baixo). Para correlacionar estatisticamente essas variáveis, esses níveis foram graduados, respectivamente em nível 3, 2 e 1.

As variáveis relacionadas com a estrutura cognitiva empresarial (regras individuais) também são classificadas em três níveis: elevado, intermediário e baixo.

No primeiro nível, elevado, os empresários demonstram a posse dos roteiros de entrada (preocupação contra imitação, redes de negócios, acesso e posse de recursos), roteiros de aspiração (foco no processo de busca, tolerância à incerteza, perseverança nas oportunidades) e roteiros de habilidade (capacidade de diagnóstico, ajuste situacional e ajuste da capacidade à oportunidade).

No segundo nível, intermediário, os empresários apresentam deficiências em, pelo menos, um dos roteiros apresentados no item anterior.

No terceiro nível, baixo, os empresários apresentam deficiências em pelo menos dois roteiros.

A associação entre a capacidade de interpretar as instituições em função da presença de uma estrutura cognitiva empresarial permite investigar as proposições apresentadas neste trabalho. A mensuração dessas variáveis é acompanhada pelas variáveis de controle:

- Desenvolvimento de, pelo menos, um negócio que se encontre em operação: Essa variável representa a experiência do empresário em constituir um negócio.

- Período de permanência de, pelo menos, três anos à frente do negócio como principal gestor: Essa variável representa um período mínimo em que o empresário se mantém à frente do negócio.

- Experiência anterior como gestor: Estoque de conhecimentos acumulados anteriores ao negócio atual.

- Educação formal na área de negócios: Conhecimentos adquiridos em unidades educacionais na área de negócios.

- Idade: Período em o indivíduo manteve relação com o ambiente externo.

\subsection{Redução de dados}

Cada empresário foi avaliado pela capacidade de reconhecer as variáveis que compõem o ambiente institucional nas dimensões regulatória, social e normativa e cultural e cognitiva. No pilar regulatório, por exemplo, os empresários que reconheceram a relevância do sistema financeiro para desenvolver negócios e a sua facilidade ou dificuldade no acesso ao capital (qualidade das instituições) foram rotulados com nível elevado de avaliação e aderência às instituições. Os empresários que reconheceram a relevância do sistema financeiro mas não souberam explicar o seu nível de qualidade foram rotulados com nível 
intermediário e níveis inferiores foram atribuídos para os empresários que se demonstraram indiferentes ao elemento institucional.

As variáveis foram reduzidas para fatores. 0 valor médio, por exemplo, das variáveis sistema financeiro, sistema legal, sistema educacional e relações de confiança determinou o nível do pilar regulatório.

0 mesmo critério foi adotado para reduzir os dados para as variáveis relacionadas aos roteiros cognitivos. 0 valor médio, por exemplo, dos roteiros de apropriação, acesso aos recursos, redes de relacionamentos e habilidades específicas determinou o nível do roteiro de entrada de cada empresário.

\section{Análise dos dados}

A associação entre a avaliação das instituições e a estrutura cognitiva empresarial foi mensurada com o uso da análise de correlações. Mensurar as correlações possibilitou evidenciar se a variação da capacidade de avaliação e aderência institucional está associada à variação das estruturas cognitivas dos empresários dentro de um SLPI. A análise de correlações permitiu avaliar se duas variáveis compartilhavam níveis aproximados de variância e produziu as seguintes informações:

- A análise de se duas variáveis compartilham níveis aproximados de variância (relacionamento).

- A direção do relacionamento (positivo ou negativo).

- A magnitude do relacionamento entre as variáveis. Essa magnitude é determinada por indicadores que representam correlações perfeitas $(+1$ ou -1$)$; fortes (entre $+0,7$ e $+0,9$ ou entre $-0,7$ e $-0,9$ ); moderadas (entre $+0,4$ e $+0,6$ e $-0,4$ e $-0,6$ ); fracas (entre $+0,1$ e $+0,3$ e $-0,1$ e $-0,3$ ); e neutras (0).

As correlações foram calculadas com o apoio do software estatístico MINITAB. As correlações entre as variáveis são apresentadas na Tabela 3.

A interpretação dos resultados apresentados pela Tabela 3 é desenvolvida nas seções a seguir com base na capacidade de o empresário avaliar as instituições em função dos roteiros cognitivos que possui.

\subsection{Capacidade de interpretação da estrutura regulatória.}

Variações nas estruturas cognitivas dos empresários apresentaram correlações com a capacidade de avaliação da estrutura regulatória. Empresários com níveis de habilidades cognitivas elevados foram capazes de reconhecer os elementos institucionais e seus níveis de qualidade.
Tabela 3. Matriz de correlações.

\begin{tabular}{lcccc}
\hline \multicolumn{1}{c}{ Variáveis } & Regulatório & Normativo & Cultural & Aspiração \\
\hline Normativo & 0,56 & & & \\
Cultural & 0,68 & 0,73 & & \\
Entrada & 0,90 & 0,70 & 0,62 & \\
Aspiração & 0,88 & 0,75 & 0,67 & 0,93 \\
Habilidades & 0,65 & 0,87 & 0,81 & 0,78 \\
\hline
\end{tabular}

\subsubsection{Roteiros de entrada versus estrutura regulatória}

Os empresários que se demonstraram mais preocupados com a proteção dos negócios (formas de apropriação), manutenção de uma rede de relacionamentos, posse de recursos e habilidades específicas para avaliar novos negócios apresentaram uma capacidade maior para avaliar a estrutura legal. A relação entre o roteiro de entrada e a estrutura regulatória pode ser detalhada da seguinte forma:

- Apropriação: Os entrevistados com preocupação de desenvolver produtos próprios, criar coleções e que estão em processo de desenvolvimento de marca própria associaram a apropriação às regras institucionais, como proteção de marcas e patentes e também a leis para proteção de contratos e defesa da concorrência. Para esses empresários, essas leis reduzem a variação no comportamento da concorrência, principalmente a redução da concorrência predatória baseada em sonegação fiscal, preços muito baixos e imitação de coleções. Empresários com níveis inferiores de habilidade cognitiva interpretaram as leis como limitadoras da atividade individual. Por exemplo, as leis de defesa da concorrência são criadas para evitar comportamentos oportunistas e manter um padrão dentro do setor. A pressão exercida por essas leis, para os empresários com foco restrito no próprio negócio, é inibidora da expansão individual do negócio.

- Acesso e posse de recursos: As variações nesses roteiros estiveram associadas com a variação na avaliação de dois elementos institucionais. 0 primeiro, sistema educacional, refere-se à necessidade de mão de obra especializada. A capacidade de o sistema educacional oferecer mão de obra em quantidade e qualidade é destacada pelos empresários como um dos principais recursos influenciados pela estrutura regulatória. A disponibilidade de centros de formação técnica é a principal base de geração desse recurso. Esses empresários também evidenciam que a oferta de mão de obra especializada é, atualmente, um fator de restrição ao desenvolvimento local. Há uma crítica intensa quanto à forma como as organizações 
públicas lidam com essa questão. Os empresários argumentam que os custos para desenvolver mão de obra são elevados e que os centros técnicos locais são extremamente ineficientes. Alguns entrevistados citam exemplos de cancelamento de pedidos por atrasos e problemas de qualidade devidos à escassez de mão de obra, enquanto o centro técnico local apresenta ociosidade. Tentativas de modificar as regras pela iniciativa empresarial têm sido vetadas pelos representantes do sistema educacional local.

0 acesso a fontes de financiamento é o segundo recurso relevante destacado pelos entrevistados. Custos, prazos e burocracia são apontados como indicadores de qualidade do ambiente regulatório:

- Habilidades específicas na avaliação de negócios: As habilidades para desenvolver novos negócios como, por exemplo, análise de mercado, análise de investimentos e riscos é relacionada à experiência e ao acesso à educação formal na área de negócios. Os empresários argumentam que as suas habilidades aumentaram com acesso aos cursos de graduação e pós-graduação na área de negócios. Empresários associam essas habilidades à necessidade de apropriação das suas criações, mas criticam o ambiente local pela ausência de uma atmosfera educacional voltada para os negócios do vestuário. A busca pelo aperfeiçoamento é um desafio individual.

Variações nos roteiros de aspiração apresentaram correlações com a capacidade de avaliar o ambiente regulatório.

\subsubsection{Roteiros de aspiração versus a estrutura regulatória}

Os roteiros de aspiração representam a busca de oportunidades, tolerância ao risco e motivação pela oportunidade de negócios. Esses roteiros representam a capacidade de os empresários manterem o foco no desenvolvimento dos negócios frente a incertezas, riscos e obstáculos que são inerentes à atividade empresarial. Variações nas estruturas cognitivas estão associadas à avaliação que os empresários fazem da confiança na estrutura legal. A política econômica, o nível de corrupção e as declarações de intenções do governo são reconhecidos como elementos que interferem no processo de busca, elevam a incerteza e produzem obstáculos para o desenvolvimento de novos negócios. A confiança está associada diretamente à visão de longo prazo dos empresários sobre a estrutura legal.

\subsubsection{Roteiros de habilidade versus a estrutura regulatória}

A capacidade de diagnosticar as oportunidades de mercado, aplicar a experiência em novos contextos e ajustar as capacidades às oportunidades é influenciada também pelo nível de confiança dos entrevistados na estrutura legal. A ambiguidade e as experiências negativas provenientes do ambiente regulatório interferem nas decisões de efetivação de novos negócios. Os empresários associam alguns fracassos empresariais à imaturidade institucional passada.

\subsection{Capacidade de interpretação da dimensão social e normativa}

A dimensão social e normativa reflete as crenças e os valores compartilhados por uma comunidade ou sociedade (Amato Neto, 2009). Essa dimensão é avaliada dentro do contexto local. Variações nas estruturas cognitivas apresentaram correlações com a dimensão social e normativa.

\subsubsection{Roteiros de entrada versus dimensão social e normativa}

As decisões empresariais relacionadas à entrada em novos empreendimentos e a sua influência da dimensão normativa apresentam os seguintes desdobramentos:

- Proteção de ideias: Uma reputação ou identidade regional é reconhecida pelos empresários como relevante para proteger os negócios. Os empresários ainda recordam que enquanto "capital nacional do jeans" a cidade tinha a preferência dos consumidores finais, lojistas, varejistas e grandes empresas do setor de vestuário. A expansão de microempresas na última década com foco mais na ação individual e pouca preocupação com a tradição local contribuiu para a perda da reputação construída durante a década de 1980. A valorização da tradição como forma de apropriação é destacada pelos empresários com níveis elevados de roteiros cognitivos.

- Acesso e posse de recursos: 0 compartilhamento de recursos produtivos e tecnológicos é apontado pelos empresários como uma vantagem competitiva para as empresas locais. Os entrevistados informaram que alguns recursos são desenvolvidos de forma redundante como, por exemplo, equipamentos de lavagem do jeans. Esses investimentos redundantes dificultam a obtenção de economia de escala. Empresários com níveis de roteiros cognitivos elevados associam os custos e a qualidade dos recursos à capacidade de cooperação dos agentes locais.

- Redes de relacionamento: Contatos, relações, troca de experiências e compartilhamentos de informações também são reconhecidos como relevantes para o desenvolvimento de novos negócios. Para os empresários com níveis inferiores nos roteiros, o compartilhamento das informações pode favorecer a concorrência local e deve ser evitado. Exemplos 
contrários são evidenciados por empresários com níveis elevados nos roteiros cognitivos. Um pequeno grupo de empresas opera atualmente dentro dos princípios da manufatura enxuta, após um processo de socialização do conhecimento com uma das confecções da cidade.

- Habilidades específicas: A socialização do conhecimento reflete-se nas habilidades de os empresários gerenciarem e explorarem novos negócios. 0 êxito com as práticas de manufatura enxuta facilita o acesso a novos contratos devido ao aumento na confiabilidade dos processos. 0 pequeno grupo de empresários com níveis de roteiros elevados encontra-se em processo de desenvolvimento de marcas próprias. Esse processo, atualmente, é dependente da socialização do conhecimento com uma empresa local que constituiu uma rede de lojas com marca própria no interior do estado de São Paulo.

\subsubsection{Roteiros de aspiração versus dimensão social e normativa}

A variação nos roteiros de aspiração também apresenta correlação com a dimensão normativa. Os empresários reconhecem que o processo de busca, a manutenção da motivação e a percepção dos riscos são influenciados pela relação com as demais empresas locais. Os empresários argumentam que, enquanto capital nacional do jeans, as empresas locais tinham foco na qualidade e na alta costura. Esse foi um período em que um padrão compartilhado assegurou a vantagem competitiva das empresas locais.

\subsubsection{Roteiros de habilidades versus dimensão social e normativa}

0 ambiente normativo cria mecanismos de autorreforço sobre as decisões empresariais. As crenças compartilhadas resultam da socialização do conhecimento acumulado dos empresários. A transferência do conhecimento e o processo de aprendizagem reforçam as habilidades individuais. Essas habilidades resultam em uma reputação local difícil de ser copiada por empresas localizadas em outro espaço geográfico. Os entrevistados evidenciam que o fim da tradição local foi determinado pela incapacidade de os empresários manterem o foco na qualidade mesmo com as pressões por custos baixos. Um dos empresários argumenta:

[...] interpretamos mal a pressão por custos e não soubemos nos adaptar. Deveríamos manter nosso padrão de qualidade e introduzir novas formas de produção, informatizar, qualificar nosso pessoal e buscar novos clientes, mas a decisão de muitas empresas locais foi voltada para cortar custos, baixos salários, redução de benefícios e finalmente uma competição predatória.

\subsection{Capacidade de interpretação da dimensão cultural-cognitiva}

As questões culturais referem-se às práticas enraizadas na sociedade ou aos padrões de comportamento que estão no inconsciente dos indivíduos. Hofstede (1980) e Hofstede et al. (2010) reproduzem na sociedade brasileira uma pesquisa sobre fatores culturais que afetam os negócios. Os empresários que apresentaram níveis mais elevados de habilidade cognitiva também apresentaram uma capacidade maior de avaliação da dimensão cultural e cognitiva das instituições.

- Individualismo: Empreender um negócio é um ato individual, mas os entrevistados apontam para um equilíbrio nos extremos entre individualismo e coletividade. Enquanto o comportamento individual assegura a evolução de um empreendimento, as ações coletivas contribuem para a construção de uma reputação local. Esses empresários identificam uma tendência ao individualismo na região.

- Intolerância à incerteza: A necessidade de regras claras e bem definidas é um dos pontos que dificultam o processo de cooperação entre as empresas locais. 0 desenvolvimento do SLPI envolve um processo evolutivo e de aprendizagem. Os resultados são difíceis de projetar com precisão. A falta de habilidade para lidar com as incertezas apresentada pelos empresários com algum deficit nos roteiros cognitivos reduz as chances de adesão desses empresários ao projeto do SLPI.

- Orientação para o longo prazo: Os entrevistados ressaltam que a preocupação com a manutenção da tradição (uma orientação para o curto prazo) é valorizada somente pelos empresários que acreditam em uma "marca região".

- Distância do poder: A participação do poder público nas atividades de empreendedorismo é destacada apenas pelos empresários que acreditam que o poder, por intermediação das políticas públicas, pode ser distribuído pela sociedade.

As variáveis que representam a dimensão cultural cognitiva apresentam uma interdependência com a dimensão normativa. Existe a necessidade de equalizar a relação entre individualismo e coletividade, compreender que novas oportunidades estão acompanhadas de incertezas, que a reputação de uma região (tradição) contribui para a consolidação de uma "marca região" e que as políticas públicas são instrumentos de redução da desigualdade social e econômica. A combinação desses quatro fatores 
está associada à dificuldade de moldar um ambiente normativo.

\subsection{As estruturas cognitivas e as variáveis de controle}

As variáveis de controle foram determinadas para evitar a influência de outras variáveis sobre a correlação entre as regras individuais e as regras sociais. A variação no nível dos roteiros cognitivos apresentou as seguintes correlações com as variáveis de controle:

- Houve forte correlação positiva $(+0,70)$ entre a presença dos roteiros e o tempo de permanência dos empresários à frente dos negócios. Essa correlação reforça que os roteiros são dependentes do processo de aprendizagem.

- A idade apresentou um coeficiente de correlação moderada $(+0,63)$. Empresários com estruturas cognitivas mais maduras estão dentro de uma faixa etária mais elevada.

- A educação formal apresenta, também, uma correlação moderada $(+0,57)$. 0 tempo de educação formal é maior para os empresários com estruturas ou roteiros cognitivos mais elevados.

- Há, também, uma correlação moderada entre tempo de atuação da empresa na região e a estrutura cognitiva desses empresários $(+0,60)$.

- Não houve correlação significativa para a variável experiência anterior como gestor de negócios $(+0,25)$.

As variáveis de controle indicam que o tempo de atuação como empresário, educação formal e idade contribuem para o processo de aprendizagem e desenvolvimento de habilidades cognitivas dentro do domínio empresarial. As variações nos roteiros cognitivos apresentaram correlações com a variação na capacidade de avaliar os elementos institucionais e a respectiva qualidade. Nesse caso, as proposições 1 e 2 foram possíveis de ser evidenciadas.

Em situações que as instituições apresentaram restrições sobre o desenvolvimento de novos negócios, empresários com níveis de cognição mais elevados relataram ações para modificar a estrutura atual. Para esse caso, há evidências para validar a proposição 3.

\section{Considerações finais}

Sistemas locais de produção e inovação em estágios embrionários dependem da criação de um ambiente institucional responsável pela coordenação das ações dos agentes locais. As regras, normas e crenças que representam as dimensões regulatória - social e normativa - e cultural e cognitiva precisam ser aceitas e alinhadas às regras individuais. 0 desenvolvimento do empreendedorismo depende, de um lado, das instituições que canalizam a atividade empreendedora e, do outro, dos roteiros cognitivos que indicam a capacidade de o empresário desenvolver novos negócios.

Empresários com níveis mais elevados de roteiros cognitivos evidenciaram uma capacidade maior de reconhecer o papel dos elementos institucionais e o seu nível de qualidade e, também, capacidade de propor e implementar mudanças quando as instituições restringem as suas ações. Empresários com níveis inferiores de habilidades cognitivas precisam de um processo de comunicação institucional diferenciado, com objetivos e regras bem definidas e, principalmente, suporte no desenvolvimento dos negócios. Níveis inferiores de estruturas cognitivas foram evidenciados em empresários com pouca ênfase na proteção de ideias, tolerância à incerteza e limitações na manutenção do processo de busca.

Essas evidências podem implicar em dificuldades para estimular a atividade empreendedora a partir de mudanças institucionais, uma vez que esses empresários possuem um foco maior na prevenção em detrimento da promoção dos recursos.

\section{Referências}

Acs, Z. J., Desai, S., \& Hessels, J. (2009). Entrepreneurship, economic development and institutions. Small Business Economics, 31(2), 219-234.

Aidis, R., Estrin, S., \& Mickiewicz, T. M. (2009). Entrepreneurial entry: Which institutions matter? Bonn: Institution for the Study of Labor. Discussion Paper n. 4123. Retrieved from http://eprints.ud.ac.uk/17685/1/17685.pdf.

Anderson, J. R. (1993). Problem solving and learning. American Psychologist, 48(1), 35-44. http://dx.doi. org/10.1037/0003-066X.48.1.35

Alexander, E. R. (2004). Institutional transformation and planning: from institutionalization theory to institutional design. Planning Theory, 3(5), 209-223.

Amato Neto, J. (2009). Gestão de Sistemas Locais de Produção e Inovação. São Paulo: Editora Atlas.

Amato Neto, J. (2005). Redes Entre Organizações: Domínio do Conhecimento e da Eficácia Operacional. São Paulo: Editora Atlas.

Baumol, W. (1990). Entrepreneurship: productive, unproductive and destructive. Journal of Political Economy, 98(5), 893-922. http://dx.doi. $\operatorname{org} / 10.1086 / 261712$

Baron, R. A. (2004). The Cognitive Perspective: A valuable Tool for Answering Entrepreneurship's Basic "Why" Questions. Journal of Business Venturing, 19(4), 221239. http://dx.doi.org/10.1016/S0883-9026(03)00008-9

Bello, D. C., Lohtia, R., \& Vinita, S. (2004). An institutional analisys of supply chain innovations in global marketing channels. Industrial Marketing Management, 33(6), 5764. http://dx.doi.org/10.1016/j.indmarman.2003.08.011 
Billet, S. (1996). Situated learning: bridging sociocultural and cognitive theorising. Learning and Instruction, 6(3), 263280. http://dx.doi.org/10.1016/0959-4752(96)00006-0

Belussi, F., Samarra, A., \& Sedita, S. R. (2008). Industrial districts evolutionary trajectories: localized learning diversity and external growth. In Proceedings of the 25th Conference on Entrepreneurship and Innovation - Organizations, Institutions, Systems and Regions, Copenhagen.

Bowen, H. P., \& De Clercq, D. (2008). Institutional context and the allocation of entrepreneurial effort. Journal of International Business Studies., 39(4), 747-767. http:// dx.doi.org/10.1057/palgrave.jibs.8400343

Bruton, G. D., Ahlstrom, D., \& Ll, H. (2010). institutional theory and entrepreneurship: where are we now and where do we need to move in the future? Entrepreneurship: Theory and Practice, 34(6), 335-347.

Busenitz, L. W. (2007). Progress in understanding entrepreneurial behavior. Strategic Entrepreneurship Journal, 1,(4), 183-185. http://dx.doi.org/10.1002/sej.5

Busenitz, L. W., \& Lau, C. (1997). A Cross-Cultural Cognitive Model of New Venture Creation. Entreneurship: Theory and Practice, 7.

Corbett, A. C., \& Hmieleski, K. (2007). The conflicting cognitions of corporate entrepreneurs. Entrepreneurship: Theory and Practice, 32(2), 105-339.

DiMaggio, P. J., \& Powell, W. W. (1983). The iron cage revisited: Institutional isophormism and collective rationality in organizational fields. American Sociological Review, 48(3), 147-160. http://dx.doi. org/10.2307/2095101

Glaser, R. (1990). The reemergence of learning theory within instructional research. American Psychologist, 45(1), 2939. http://dx.doi.org/10.1037/0003-066X.45.1.29

Greif, A. (2006). Institutions and the path to the modern economy. Lessons from medieval trade. California: Cambridge University Press. http://dx.doi.org/10.1017/ CB09780511791307

Hessels, J., Van Gelderen, M., \& Thurik, R. (2008). Entrepreneurial aspirations, motivations and their drives. Small Business Economics, 31(3), 1007-1127. http:// dx.doi.org/10.1007/s11187-008-9134-x

Hofstede, G. (1980). Culture's Consequences: International Differences in Work Related Values. Beverly Hills: Sage Publications.

Hofstede, G., Devilaal, A. V. G., Tanure, B., \& Vinken, H. (2010). Comparing Regional Cultures within a Country: Lessons from Brazil. Journal of CrossCultural Psychology, 41(7), 336-351. http://dx.doi. org/10.1177/0022022109359696

Holm, P. (1995). The dynamics of institutionalization: transformation process in Norwegian fisheries. Administrative Science Quaterly, 40(4), 398-422. http:// dx.doi.org/10.2307/2393791

Leandro, C. R., \& Amato Neto, J. (2007). Building competence to innovate through cooperation networks: A project for Sorocaba metropolitan region. In Proceedings of the 14th International Annual European Operations Management Society (EUROMA) Conference.

Leddo, J., \& Abelson, R. P. (1986). The nature of explanations. In J. A. Galambos, R. P. Abelson \& J. B. Black (Eds.), Knowledge Structures. New Jersey: Hillsdale, Lawrence Erlbaum and Associates.
Lepak, D. P., Smith, K. G., \& Taylor, S. M. (2007). Value Creation and Value Capture: A Multilevel Perspective. Academy of Management Review, 32(1), 180-194. http://dx.doi.org/10.5465/AMR.2007.23464011

Lim, D. S. K., Morse, E. A., Mitchell, R. K., \& Seawright, K. K. (2010). Institutional Environment and Entrepreneurial Cognitions: A Comparative Business System Perspective. Entrepreneurship: Theory and Practice, 37(2), 237-249.

Manolova, T. S., Eunni, R. V., \& Gyoshev, B. S. (2008). Institutional Environments for Entrepreneurship: Evidence from Emerging Economies in Eastern Europe. Entrepreneurship Theory and Practice, 32(3), 234, 345.

Marquis, C., \& Battilana, J. (2009). Acting Globally but Thinking Locally? The Enduring Influence of Local Communities on Organizations. Research In Organizational Behavior, 29(5), 283-302. http://dx.doi. $\operatorname{org} / 10.1016 /$ j.riob.2009.06.001

Meyer, J. K., \& Rowman, B. (1991). Institutionalized organizations: formal structure as myth and ceremony In W. W. Powell, J. P. DiMaggio (Eds.), The new institutionalism in organizational analisys. Chicago: University of Chicago Press.

Meyer, K., Estrin, S., Bhaumik, S. K., \& Peng, M. W. (2009). Institutions, Resources and Entry Strategies in Emerging Economies. Strategic Management Journal, 30(1), 6180. http://dx.doi.org/10.1002/smj.720

McGee, J. E., Peterson, M., Mueller, S. L., \& Sequeira, J. M. ( 2009). Entrepreneurial Self-Efficacy: Refining the Measure. Entrepreneurship: Theory and Practice, 30(2), 70-86.

Mitchell, R. K., Seawright, K. K., \& Morse, E. A. (2000). CrossCultural Cognitions and The Venture Creation Decision. Academy of Management Journal, 43(5), 180-197, http://dx.doi.org/10.2307/1556422

Mitchell, R., Busenitz, L., Bird, B., Gaglio, C. M., Mcmullen, J., Morse, E., \& Smith, B. (2007). The Central Question in Entrepreneurship Cognition Research. Entrepreneurship: Theory and Practice, 31(1), 1-27.

North, D. (1990). Institutional Change and Economic Performance. New York: Norton. http://dx.doi. org/10.1017/CB09780511808678

Peng, M. W., Wang, D. Y. L., \& Jiang, Y. (2008). An Institution- Based View of International Business Strategy: A Focus on Emerging Economies. Journal of International Business Studies, 39(8), 920-936. http:// dx.doi.org/10.1057/palgrave.jibs.8400377

Scott, W. R. (2007). Institutions and Organizations: ideas and interests. Thousand Oaks: Sage.

Shepsle, K. A., \& Bonchek, M. S. (1996). Analyzing politics: rationality, behavior and institutions. New York: W.W. Norton.

Schumpeter, J. A. (1934). Teoria do Desenvolvimento Econômico. Rio de Janeiro: Editora Fundo de Cultura.

Smith, B. J., Mitchell, J. R., \& Mitchell, R. K. (2009). Entrepreneurial Scripts and The New Transaction Commitment: Extending EIPT Approach to Entrepreneurial Cognition research. Entrepreneuruship: Theory and Practice, 34(4), 361-383.

Stephan, U., \& Uhlaner, L. M. (2010). Performance-based versus supportive culture: A cross-natinal study of descriptive norms an entrepreneurship. Journal of International Business Studies, 41(2), 1347-1364. http:// dx.doi.org/10.1057/jibs.2010.14 


\title{
The institutionalization process of local systems of production and innovation: a cognitive approach
}

\begin{abstract}
The evolution of local systems of production and innovation (LSPI) is followed by the institutionalization of the system to coordinate the actions of entrepreneurs and other local agents. The process of alignment between institutions (social rules) and cognitions (individual rules) is determined by impasses and contradictions, once changes in both dimensions do not occur simultaneously. This article analyzes how the level of entrepreneurial cognitive ability influences the capacity of entrepreneurs for interpreting institutions. This research, developed in a garment district of Indaiatuba with twenty entrepreneurs, proposes a methodology, within an entrepreneurial domain, to measure the level of entrepreneurial cognitive ability and the capacity of entrepreneurs to read and interpret institutions. There are two main findings. First, there is evidence that entrepreneurs within a LSPI have different levels of cognitive structures. Second, these differences are correlated with the capacity of entrepreneurs to read, interpret and adopt institutions.
\end{abstract}

Keywords

Institutions. Entrepreneurship. Entrepreneurial cognition. Local system of production and innovation. 